used to review types of poisoning, culprit mushrooms, and available treatments.

Results.-Over the last 17 years 127,279 cases (7487/year) of mushroom exposure, mostly ingestion, have been reported. Cases are most frequently unintentional $(83 \%, P<.001)$, cause no or only minor harm $(86 \%, P<.001)$, occur in children $<6$ years old $(62 \%, P<.001)$. Approximately 665 (39/year) exposures have resulted in major harm. Fifty (2.9/year) fatalities have occurred, mostly $(83 \%)$ from cyclopeptide-containing mushrooms ingested by older adults (mean age $61.0 \pm 18.5$ years).

Conclusions.-The National Poison Data System recognizes 7 distinct groups of poisoning, although many more are likely to exist. The vast majority of ingestions result in no or minor harm, although some groups, like cyclopeptides, ibotenic acid, and monomethylhydrazine, can be deadly.

\section{Cardiovascular Implantable Electronic Devices and Scuba Diving: from Cardiac Electrophysiol- ogy Point of View}

Anthony Chang

Virginia Heart, Falls Church, VA, USA

Introduction.-People with cardiovascular implantable electronic devices (CIEDs) may wish to participate in scuba diving (SD). To best advise these patients, health care providers must have the necessary knowledge about CIEDs, including the impact of hyperbaric pressure, the indications, and the individual characteristics and limitations of different CIEDs.

Objective.-To review literature on CIEDs and SD and to present the latest information on CIEDs relating to hyperbaric pressure and the current CIEDs guidelines relevant to diving.

Methods.-The literature on CIEDs and SD, including technical documents of CIEDs from 4 major manufacturers (Biotronik, Boston Scientific, Medtronic, and St. Jude), and practice guidelines of CIEDs were reviewed. Testing methods, results, differential effects on the types of CIEDs (pacemaker, defibrillator, and loop recorder), and case reports were obtained from the manufacturers.

Results.-Existing literature on hyperbaric pressure and CIEDs contain outdated information. Recommendations regarding CIEDs and SD lack specifics on different types of CIEDs and do not address the underlying heart conditions. Indications for CIEDs range from mild symptoms to lifethreatening conditions that will limit SD. CIED test pressure varies from 3 to 7 ATM among manufacturers. Casing deformation can occur, but no component integrity or electronic function is compromised. Devices using piezoelectric sensors may cause undesired heart rate acceleration. No case of device malfunction related to diving or hyperbaric pressure has been reported to the manufacturers during the past 10 years. Some CIEDs are capable of wireless transmission using cellular and WiFi communicators best suited for remote monitoring.

Conclusions.- The underlying cardiac conditions should be the most important determinant when advising people with CIEDs who wish to engage in SD. Current generations of CIEDs function well under tested pressures. Knowing the individual characteristics and limitations of different CIEDs will help in selecting specific devices before implantation and in advising divers with existing devices.

\section{Death on the Dome: Epidemiology of Recrea- tional Deaths on Half Dome in Yosemite National Park}

Gregory Richardson; Susanne Spano

University of California San Francisco Fresno, Fresno, CA, USA

Introduction.-Half Dome is a popular destination in Yosemite with inconsistent public reporting of recreational mortality. The highest number of deaths in a single source is 20 .

Methods.-Multimedia sources describing deaths involving the cables, summit, climbing, or base jumping were included. Deaths occurring on trails were excluded.

Results.-Twenty-nine deaths occurred on Half Dome, with 2 additional deaths likely. The earliest was in 1930. The rate of 1 to 2 deaths per decade ended in the 1980 s, which saw 8 fatalities. The 2000s had 9 fatalities. This decade has 4 deaths currently. The typical profile is a 32-year-old hiking man (only 4 women were reported); the median age is 27 years (range 16-86). Hikers $(12 ; 39 \%)$ predominated, followed by 9 climbers $(29 \%)$. The most common mechanism was climbing incidents related to lost anchors (5), free climber falls (3), and head injury from rock fall (1). Fatal hiker falls from the cables (7) were occasionally weather related (3), and 71\% (5) occurred on descent. Lightning caused 3 summit deaths and a cablerelated death. Medical mortality, in 3 hikers, was attributed to cardiac causes (2) and altitude illness (1). There were 8 suicides (29\%). Base jumping fatalities (2) resulted from chute malfunction and wind slamming a jumper into Half Dome's face.

Conclusions.-We identified 31 Half Dome deaths, the highest published. A minority of deaths were attributable to unfavorable weather or unskilled hikers, despite this being a popular assertion. Climber accidents, often unpublicized, challenge the assumption that this increasingly popular sport is safe. These findings urge a particular educational focus on accidents during descent of the cables, a renewed focus on suicide prevention, and prompt consideration of permitting climber access to reduce mortality on Half Dome. 